\title{
Case report: a peculiar glomerulopathy in a patient suffering from nephrotic syndrome
}

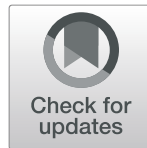

Fabian Wöstmann ${ }^{1}$, Roman-Ulrich Müller ${ }^{1,2}$, Heike Göbel ${ }^{3}$, Thomas Benzing ${ }^{1,2}$, Jan U. Becker ${ }^{3^{*}}$ and

Malte P. Bartram ${ }^{1 *}$ (D)

\begin{abstract}
Background: Podocyte infolding glomerulopathy $(\mathrm{PIG})$ is a rare histopathologic finding with global infolding of the podocytes into the glomerular basement membrane (GBM), accompanied by microstructures underneath. Described in 2002 for the first time, PIG was proposed as a new pathological entity in 2008 based on the largest case series so far. Yet all of the described cases derive from Asian countries. We report a case from Germany fulfilling the diagnostic criteria of PIG. Considering the scarcity of data on this entity especially in Western countries, collecting cases like ours and multicentric meta-analyses will be crucial to obtain a better understanding of PIG, its causes, clinical course and potential treatment options.
\end{abstract}

Case presentation: A 56-year-old Caucasian woman with a history of rheumatoid arthritis (RA), no other comorbidities and no known renal disease was admitted to the hospital with acute kidney injury (AKI) and nephrotic syndrome. Physical examination was unremarkable except for anasarca. Renal ultrasound revealed no abnormalities. Laboratory and urine analyses were consistent with the nephrotic syndrome and renal failure. Serological studies regarding ANA, ANCA, anti-PLA2R autoantibodies, complement, virus infections, immunofixation and quantitative light chain analysis were unremarkable. A renal biopsy was performed. Light microscopic examination showed flattened tubular epithelium consistent with acute tubular damage, no infiltrates and unremarkable glomeruli except diffuse and global holes in the GBM (Fig. 1a) and negative staining for immunoglobulin heavy-chains, light-chains and complement split products. Electron microscopy revealed a rare correlate for these holes: global peculiar infolding of podocyte cytoplasm into the GBM. Most of these infoldings were accompanied by condensation of the GBM underneath. No such condensation or electron dense deposits were found without these infoldings or outside the GBM.

Conclusion: Here we report the first case of PIG outside of Asia. Since there are only few reports about this specific finding, we feel there is a need to share information in an attempt to accumulate knowledge about this possible new entity and potential treatment options.

Keywords: Nephrotic syndrome, Membranous Glomerulopathy, Microspheres, Podocyte infolding, Renal biopsy

\section{Background}

PIG is a rare and peculiar finding in kidney biopsies. The largest study up to date, conducted by Joh et al. in 2008 comprised 25 Patients, all from Japan [1]. Outside of Japan only two cases have been published by now, one in South Korea [2] and another from India [3].

*Correspondence: janbecker@gmx.com; malte.bartram@uk-koeln.de ${ }^{3}$ Institute of Pathology, University Hospital of Cologne, Cologne, Germany ${ }^{1}$ Department II of Internal Medicine and Center for Molecular Medicine Cologne, University of Cologne, Faculty of Medicine and University Hospital Cologne, Cologne, Germany

Full list of author information is available at the end of the article
Accompanying clinical findings and the age of onset show a wide variation. Most patients so far have been female and there was a noticeable association with autoimmune disorders, especially systemic lupus erythematosus (SLE) and other collagen diseases [1]. Also one case associated with multiple myeloma in a Japanese patient was described [4] The Japanese consensus recommends a clinical sub-classification depending on whether PIG is associated with collagen disease or not [1]. Accordingly, our Patient would be classified as PIG type A, collagen disease-associated.

Altogether, PIG appears to be a histopathological pattern common to a number of different causes. Up to 


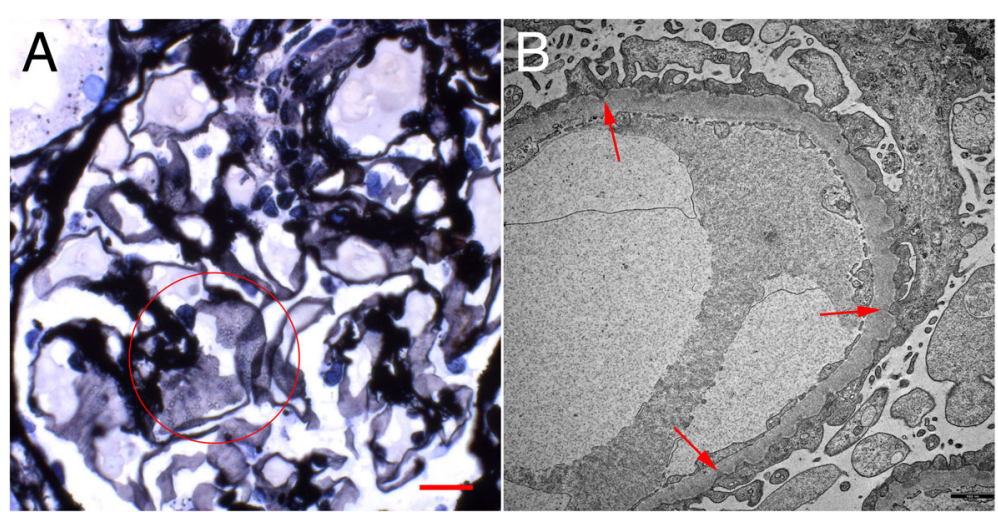

Fig. 1 a Jones silver staining shows holes in the glomerular basement membrane (circle). Original magnification $\times 630$, scale bar represents $50 \mu \mathrm{m}$. $\mathbf{b}$ Electron microscopy shows the ultrastructural correlate to these holes: infolding of the podocyte cytoplasm into the glomerular basement membrane, most of them with underlying densities (red arrows). This finding is characteristic for the podocyte infolding glomerulopathy (PIG). Transmission electron microscopy, original magnification $\times 10000$, scale bar represents $1000 \mathrm{~nm}$ )

now, there is discussion how this very rare nephropathological entity translates into a clinical diagnosis and what is the preferred treatment. As to the clinical course and light microscopic findings, PIG may be difficult to distinguish from certain forms of membranous nephropathy (MN) [5]. In our case both would be well in line with an atypical form of MN. However, neither immunohistochemistry nor EM revealed the typical immune complexes of MN.

We hope this case description, the first from a Western country, contributes to the understanding of this rare disease.

\section{Case presentation}

A 56 year old obese Caucasian woman with a 12 year history of rheumatoid arthritis (RA), no other comorbidities and no known renal disease was transferred to our hospital with acute kidney injury and nephrotic syndrome.

The patient had been well until 11 days prior to admission when she experienced joint pain. She continued her regular RA medication with certolizumab and NSAID. Within the following days the patient noted oliguria and swelling of the face and legs. On admission, the patient presented well-oriented, with normal vital parameters (blood pressure $130 / 75 \mathrm{mmHg}$, heart rate $84 / \mathrm{min}$ ) and a body weight of $87.9 \mathrm{~kg}$ (regular weight $82 \mathrm{~kg}$ ). Physical examination was inconspicuous except for anasarka and an erythematous rash with papules in the epigastric region, appearing shortly after the self-administered dose of certolizumab.

Renal ultrasound revealed no abnormalities, there were no signs of renal vein thrombosis.

Laboratory results were consistent with the clinical picture of the nephrotic syndrome and showed an increased creatinine of $4.37 \mathrm{mg} / \mathrm{dl}$, hypalbuminemia of 18 $\mathrm{g} / \mathrm{l}$ and hypercholesterinemia (LDL $301 \mathrm{mg} / \mathrm{dl}$ ). Urinary diagnostics showed glomerular proteinuria in the nephrotic range (albuminuria $6.2 \mathrm{~g} / \mathrm{g}$ creatinine, erythrocytes $35 \mathrm{U} / \mathrm{l})$. Serological studies regarding ANA, ANCA, antiPLA2R autoantibodies, complement, viral testing (HBV, HCV, HIV, Hantavirus), serum/urine immunofixation and quantitative light chain analysis returned negative.

A renal biopsy was performed. Light microscopic examination showed flattened tubular epithelium consistent with acute tubular damage, no infiltrates and unremarkable glomeruli except diffuse and global holes in the glomerular basement membrane (GBM) on Jones' silver stains (Fig. 1a) and negative staining for immunoglobulin heavy-chains, light-chains and complement split products. Electron microscopy (EM) showed the peculiar findings in Fig. 1b.

In most cases, holes in the GBM are indicative of membranous nephropathy (MN). However, MN was ruled out by negative immunohistochemistry. Instead, EM did reveal a different, exceedingly rare correlate for these holes: global peculiar infolding of the silver negative podocyte cytoplasm into the silver positive GBM [1]. Such infolding is not found in minimal change glomerulopathy. Most of these infoldings were accompanied by condensation of the GBM underneath. No such condensation or electron dense deposits were found without these infoldings or outside the GBM ruling out abortive forms of MN which can show only scant immunoglobulin and complement deposits. Moreover, the typical thinning, thickening and basket-weaving of Alport's syndrome, an important differential diagnosis, were absent.

According to the Japanese consensus definition, these findings are diagnostic for PIG. The largest study up to date comprised 25 Patients from Japan [1]. Outside of Japan only two cases have been published [2, 3]. We could not find spheroid or tubular inclusions in the GBM which accompany the infoldings in PIG type B 
and exist without these infoldings in PIG type C. Thus our case would be classified as PIG type A.

Clinical findings and the age of onset show a wide variation. Most patients were female and there was an association with autoimmune disorders, especially systemic lupus erythematosus (SLE) and other collagen diseases [1].

Up to now, there is discussion how this very rare nephropathological entity translates into a clinical diagnosis and about the preferred treatment. As illustrated in our case, the clinical course and light microscopic findings of PIG may be difficult to distinguish from certain forms of MN [5]. However, neither immunohistochemistry nor EM revealed the typical immune complexes of MN. Based on the biopsy findings and clinical pattern we considered this case as a RA-associated nephropathy with PIG. Taking into account the good response in a similar case and the rheumatic disease [3], we discontinued certolizumab and started treatment with high dose prednisone and rituximab $(2 \times 1 \mathrm{~g}$ rituximab given 2 weeks apart), afterwards the steroids were rapidly tapered.

After initiating this therapy, albuminuria (from $6.22 \mathrm{~g} /$ $\mathrm{g}$ to $2.63 \mathrm{~g} / \mathrm{g}$ creatinine) as well as serum creatinine levels (from $4.37 \mathrm{mg} / \mathrm{dl}$ to $2.81 \mathrm{mg} / \mathrm{dl}$ ) declined within 4 weeks. Two months later, there was no relevant albuminuria detectable and the serume creatinine levels remained stable around $0.9-1.0 \mathrm{mg} / \mathrm{dl}$ (Fig. 2). In addition, the patient had absolutely no complaints regarding her RA.

\section{Discussion and conclusions}

So far most reports about PIG have speculated on the pathogenesis and etiology of the infolding and underlying microstructures accompanying the infoldings in PIG type B and C. Still there is no consensus about the origin of the infoldings or the underlying microstructures, animal models of PIG are still missing. Hepatitis B virus [6], complement activation [5], villous cytoplasmic protrusions of endothelial cells [1], or a reaction to podocyte injury secondary to a kidney injury [7] have been suggested as the cause of the microstructure formation in the GBM. Nevertheless, so far none of the above mentioned was proven to be a common feature of all the known cases of podocyte infolding.

Podocytic infolding per se can have several causes as shown by Masuda et al. in a series of 126 biopsies with membranous nephropathy [6]. $77.8 \%$ of the cases showed occasional podocytic infolding. However global and diffuse podocytic infolding as observed in our cases was very rare and only found in one of the 126 cases [6]. Certainly Alport's syndrome is in the list of differential diagnoses. The clinical course in our patient and the lack of more typical findings argue against this differential diagnosis; in case of doubt genetic testing should be considered.

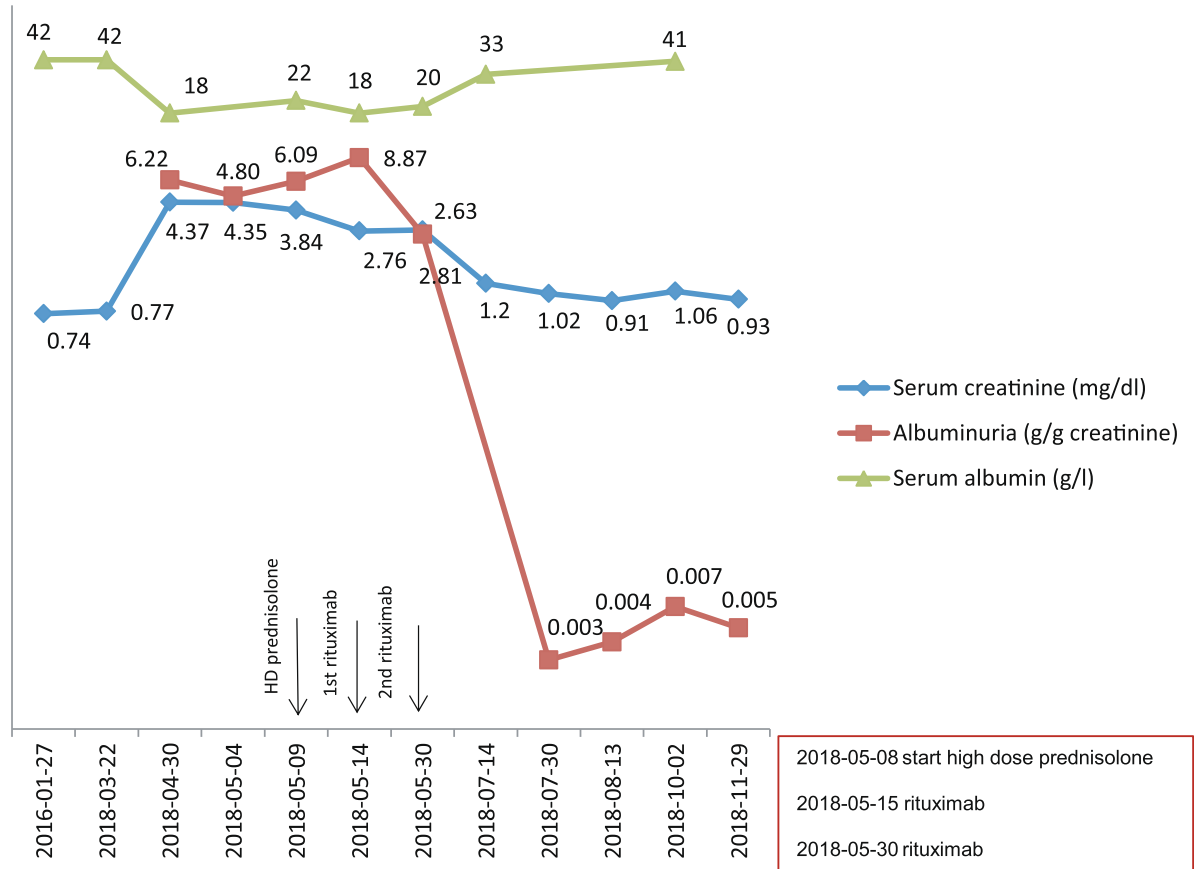

Fig. 2 Graph showing the course of serum creatinine, albuminuria and serum albumin after onset of the disease and after initiation of the very effective treatment with prednisolone and rituximab. Note the logarithmic scale of the $y$-axis 
The patient described in this report presented with acute kidney injury. From the cases published so far, only around $25 \%$ had increased serum creatinine levels $[1-4,8]$. In our case the intake of NSAIDs may have contributed to the development of AKI.

With the etiology and contibuting factors still unclear and a varied clinical presentation PIG should be considered a pattern of injury rather than an etiologically defined diagnostic entity, much like podocyte foot process effacement or focal and segmental glomerulosclerosis (FSGS). In line with the published literature we believe that the RA might be responsible - through an unknown mechanism - for the renal disease of our patient. Reviewing the published cases, none has been associated with a TNF-alpha inhibitor. Still - considering the scarcity of data - we cannot exclude a causal link. We hope that our case report will alert nehrologists and nephropathologists to this rare diagnostic entity and will stimulate larger case collections required to better define the etiology, diagnostic criteria, clinical associations, prognosis and treatment options for PIG.

Since many cases are connected to collagen and other rheumatic diseases we would like to emphasise the importance of a renal biopsy for these patients.

Regarding the literature most patients with the nephropathological pattern of a podocyte infolding glomerulopathy (PIG) in the kidney biopsy have been treated with steroids $[1,4,7,8]$, sometimes additional immunosuppressants like mycophenolate mofetil (MMF) or rituximab were utilised. To avoid long term high dose steroid treatments and considering the frequent coexistence of autoimmune diseases, it might be helpful to initiate a steroid sparing treatment early on. In our case the treatment with rituximab was very effective in reducing the proteinuria and improving the renal function as well as improving the rheumatoid arthritis.

\begin{abstract}
Abbreviations
AKl: Acute kidney injury; ANA: Antinuclear antibodies; ANCA: Anti-neutrophil cytoplasmatic antibodies; Anti-PLA2R: Anti-Phospholipase A2 receptor; FSGS: Focal and segmental glomerulosclerosis; GBM: Glomerular basement membrane; MMF: Mycophenolate mofetil; MN: Membranous nephropathy; NSAID: Nonsteroidal anti-inflammatory drugs; PIG: Podocyte infolding glomerulopathy; RA: Rheumatoid arthritis; SLE: Systemic lupus erythematosus
\end{abstract}

\section{Acknowledgments}

We thank the patient for giving her consent to use the results obtained by the kidney biopsy for research and clinical research purposes. We thank the members of our departments for helpful discussions. R.-U.M was supported by the Nachwuchsgruppen.NRW program of the Ministry of Science North Rhine Westfalia (MIWF, to R.-U.M.) and the German Research Foundation (KFO329, MU3629/3-1).

\section{Authors' contributions}

F.W., T.B., R-U.M. and M.P.B. treated the patient. H.G. and J.U.B. performed the histopathological analysis of the kidney biopsy. F.W., R-U.M., J.U.B. and M.P.B. wrote the manuscript. All authors read and approved the final version of the manuscript.

\section{Funding}

R.-U.M was supported by the Nachwuchsgruppen.NRW program of the Ministry of Science North Rhine Westfalia (MIWF, to R.-U.M.) and the German Research Foundation (KFO329, MU3629/3-1).

\section{Availability of data and materials}

All the data relevant to this report are included in the manuscript.

\section{Ethics approval and consent to participate}

Not applicable

\section{Consent for publication}

The patient gave written informed consent to use the results obtained by the kidney biopsy for research and clinical research purposes prior to the kidney biopsy. Furthermore the patient gave written informed consent for publication of this report and the associated images.

\section{Competing interests}

The authors declare no conflict of interest. The results presented in this paper have not been published previously in whole or part anywhere else.

\section{Author details}

${ }^{1}$ Department II of Internal Medicine and Center for Molecular Medicine Cologne, University of Cologne, Faculty of Medicine and University Hospital Cologne, Cologne, Germany. ${ }^{2}$ CECAD, University of Cologne, Faculty of Medicine and University Hospital Cologne, Cologne, Germany. ${ }^{3}$ Institute of Pathology, University Hospital of Cologne, Cologne, Germany.

Received: 22 January 2019 Accepted: 19 July 2019

Published online: 22 August 2019

\section{References}

1. Joh K, Taguchi T, Shigematsu H, Kobayashi $Y$, Sato H, Nishi S, Katafuchi R, Nomura S, Fujigaki Y, Utsunomiya Y, Sugiyama H, Saito T, Makino H. Proposal of podocytic infolding glomerulopathy as a new disease entity. A review of 25 cases from nationwide research in Japan. Clin Exp Nephrol. 2008;12:421-31.

2. Kwon $\mathrm{KW}$, Jeong $\mathrm{HJ}$, Lee JH. Podocytic infolding glomerulopathy. A case report. Ultrastruct Pathol. 2016;40:374-7.

3. Matthai SM, Mohapatra A, Mathew AJ, Roy S, Varughese S, Danda D, Tamilarasi V. Podocyte Infolding Glomerulopathy (PIG) in a patient with undifferentiated connective tissue disease. Am J Kidney Dis. 2018;72(1):149-53.

4. Harada M, Kamijo Y, Ehara T, Shimojo H, Shigematsu H, Higuchi M. A case of podocytic infolding glomerulopathy with multiple myeloma. BMC Nephrol. 2014;15:32.

5. Fujigaki $Y$, Muranaka $Y$, Sakakima M, Onta I, Sakao $Y$, Fujikura T, Sun $Y$, Katafuchi R, Joh K, Hishida A. Analysis of intra-GBM microstructures in a SLE case with glomerulopathy associated with podocytic infolding. Clin Exp Nephrol. 2008;12:432-9.

6. Masuda Y, Mii A, Shimizu A, Fujita E, Aki K, Ishikawa K, Ishizaki M, Sato S, Hayama N, lino Y, Katayama Y, Fukuda Y. Invagination and infolding of podocytes in glomerular basement membrane in the cases of primary membranous nephropathy. Clin Exp Nephrol. 2008;12:440-9.

7. Matsuo T, Kobayashi Y, Nemoto N, Sano T, Kamata K, Shigematsu H. A nephrotic case of vesicoureteral reflux representing focal segmental glomerulosclerosis associated with podocytic infolding lesions. Clin Exp Nephrol. 2008;12:494-500

8. Iguchi A, Sohma A, Yamazaki H, Ito T, Saeki T, Ito Y, Imai N, Osawa Y, Narita I. A case of podocytic infolding glomerulopathy with focal segmental glomerulosclerosis. Case reports in nephrology and urology. 2013;3:110-6.

\section{Publisher's Note}

Springer Nature remains neutral with regard to jurisdictional claims in published maps and institutional affiliations. 